\title{
The Quantum Mechanical Rotation Operators of Spins 5/2 to 7/2
}

\author{
Mehpeyker Kocakoç ${ }^{1}$, Recep Tapramaz ${ }^{2}$ \\ ${ }^{1}$ Department of Computer Technologies, Vocational School of İmamoğlu, Çukurova University, Adana, Turkey \\ ${ }^{2}$ Department of Physics, Ondokuz Mayıs University, Samsun, Turkey \\ Email: mkocakoc@cu.edu.tr, recept@omu.edu.tr
}

How to cite this paper: Kocakoç, M. and Tapramaz, R. (2021) The Quantum Mechanical Rotation Operators of Spins 5/2 to 7/2. Journal of Applied Mathematics and Physics, 9, 856-863.

https://doi.org/10.4236/jamp.2021.95058

Received: December 25, 2020

Accepted: May 11, 2021

Published: May 14, 2021

Copyright $\odot 2021$ by author(s) and Scientific Research Publishing Inc. This work is licensed under the Creative Commons Attribution International License (CC BY 4.0).

http://creativecommons.org/licenses/by/4.0/

\begin{abstract}
With the creation of logic gates and algorithms for quantum computers and entering our lives, it is predicted that great developments will take place in this area and important efforts are made. Spin rotation processors are quantum mechanical rotation processors and have no classic counterparts. The rotation operators of spin 1/2 are well known and can be found in related textbooks. But rotation operators of other spins greater than $1 / 2$ can be found numerically by evaluating the series expansions of exponential operator obtained from Schrödinger equation, by evaluation of Wigner-d formula or by recently established expressions in polynomial forms discussed in the text. In a previous paper, rotation operators for spins $1 / 2$ to 2 were published. In this work, explicit symbolic expressions of $x, y$ and $z$ components of rotation operators for spin 5/2,3 and $7 / 2$ are worked via exponential operator for each element of related spin operators and utilizing simple linear curve fitting process. The procedures gave out exact expressions of each element of the rotation operators.
\end{abstract}

\section{Keywords}

Rotation Operator, Quantum Information Systems, Spin, Electron Paramagnetic Resonance (EPR)

\section{Introduction}

Quantum mechanical rotation operators in explicit forms are the essential tools of microscopic systems. Wigner [1] introduced expressions of rotation matrices or Wigner-d matrices for orbital angular momenta with the basis of standard Euler angles. Real rotation operators for total angular momenta of $1 / 2,1,3 / 2$ and 2 were generated from Wigner-d formula in some classical textbooks of quantum mechanics [2]-[8]. A recently published paper on rotation operators by 
Curtright et al. (2014) and Curtright and Van Kortryk [9] [10] give rotation operator expressions in polynomial form for all angular momenta in Cartesian components. In order to find out the rotation operators in a matrix form, one has to sum up the polynomial terms given, which includes powers of related angular momentum operators.

Pulsed nuclear magnetic resonance, (pulsed-NMRnuclear magnetic resonance), pulsed electron paramagnetic resonance (pulsed-EPRElectron paramagnetic resonance) and pulsed electron nuclear double resonance (pulsedENDORElectron nuclear double resonance) spectroscopies, however, utilizes rotation operators in rotating coordinate system or laboratory coordinate system where the spins are polarized along a definite orientation by a known external magnetic field. This direction is defined as $z$ axis and a series of magnetic pulses are applied consequently along laboratory $x$ and/or $y$ axes to rotate the polarized spins around related axes [11] [12] [13] [14] [15]. Besides the spectroscopic applications, spin based quantum-computing systems in which the pulsed magnetic resonance techniques constructed the pioneering pulse arrays, utilizes rotation operators intensively in rotating Cartesian coordinate system [13] [14].

In this work explicit rotation operator expressions of angular momenta $5 / 2$ to $7 / 2$ are constructed from exponential operators given in equality below for $x, y$ and $z$ components of angular momenta for a series of angles $\theta$ between interval $\theta_{1}$ to $\theta_{N}$, and fitting the obtained values to suitable functions by linear curve fitting procedure.

\section{Rotation Operator Expression}

We start rewriting the exponential rotation operator expression,

$$
\hat{R}_{\alpha}=\exp \left(i \theta_{p} \hat{J}_{\alpha}\right), \alpha=x, y, z
$$

derived from time dependent Schrödinger equation for a rotating magnetic field pulse B1 given as $-i \hbar \frac{\partial \psi}{\partial t}=\hat{\mathcal{H}} \psi$ where $\hat{\mathcal{H}}=g \beta B_{1} \hat{J}_{\alpha}$ is pulse Hamiltonian applied to a spin system polarized along laboratory $z$ axis by an external magnetic field, where $\hat{J}_{\alpha}(\alpha=x, y, z)$ is Pauli spin matrices. $\hat{J}_{\alpha}$ can be nuclear spin $I$ or electron spin $S$, or coupled $S$ and $I$ systems, and $g$ and $\beta$ have conventional meanings. The rotation angle $\theta_{p}$ is defined for rotating pulse duration $t_{p}$ as $\theta_{p}=g \beta B_{1} t_{p} / \hbar=\omega_{p} t_{p}$.

The exponential function for nuclear spin in equation above can be rewritten by Euler expansion,

$$
R_{\alpha}=\exp \left(i \theta_{p} \hat{J}_{\alpha}\right)=\cos \left(\theta_{p} \hat{J}_{\alpha}\right)+i \sin \left(\theta_{p} \hat{J}_{\alpha}\right), \alpha=x, y, z
$$

where;

$$
\begin{aligned}
& \cos \left(\theta_{p} \hat{I}_{\alpha}\right)=\mathbb{I}-\frac{1}{2 !} \theta_{p}^{2} J_{\alpha}^{2}+\frac{1}{4 !} \theta_{p}^{4} J_{\alpha}^{4}-\frac{1}{6 !} \theta_{p}^{6} J_{\alpha}^{6}+\cdots=\sum_{n=0}^{\infty} \frac{1}{(2 n) !} \theta_{p}^{2 n} J_{\alpha}^{2 n} \\
& \sin \left(\theta_{p} \hat{I}_{\alpha}\right)=\frac{1}{1 !} \theta_{p}^{1} J_{\alpha}^{1}-\frac{1}{3 !} J_{\alpha}^{3} \theta_{p}^{3}+\frac{1}{5 !} J_{\alpha}^{5} \theta_{p}^{5}-\cdots=\sum_{n=0}^{\infty} \frac{1}{(2 n+1) !} \theta_{p}^{2 n+1} J_{\alpha}^{2 n+1}
\end{aligned}
$$


where $\mathbb{I}$ is the unit matrix with the same dimension as $J$.

Evaluation of power series given in above requires intensive calculation due to the powers of spin operator matrices. The rotation operators for spin-1/2 systems are rather easy because the elements of the powers of Pauli spin matrices are either zero or unity multiplied by a coefficient. As an example, Pauli matrices for spin-1/2 and corresponding explicit rotation operators are borrowed from textbooks [11] [12] [13] and are given in Equation,

$$
\begin{aligned}
& \hat{I}_{x}=\frac{1}{2}\left[\begin{array}{ll}
0 & 1 \\
1 & 0
\end{array}\right], \hat{I}_{y}=\frac{i}{2}\left[\begin{array}{cc}
0 & -1 \\
1 & 0
\end{array}\right], \hat{I}_{z}=\frac{1}{2}\left[\begin{array}{cc}
1 & 0 \\
0 & -1
\end{array}\right] \\
& \hat{R}_{x}(\theta)=\left[\begin{array}{cc}
c & i s \\
i s & c
\end{array}\right], \hat{R}_{y}(\theta)=\left[\begin{array}{cc}
c & s \\
-s & c
\end{array}\right], \hat{R}_{z}(\theta)=\left[\begin{array}{cc}
z & 0 \\
0 & z^{*}
\end{array}\right]
\end{aligned}
$$

where $c=\cos (\theta / 2), s=\sin (\theta / 2), z=c+i s$.

One of the ways of obtaining explicit expressions of the rotation operators for the spins greater than $1 / 2$ is two-step numerical calculation. In the first step the sine and cosine series given in powers of spin operator matrices are summed up numerically for each element of a spin matrix for angles between e.g. $0^{\circ}$ and $360^{\circ}$ with intervals of $5^{\circ}$, and with the highest possible precision. The results for each angle are found to be real and imaginary elements of rotation matrices.

In the second step, variations of each element of the rotation matrices against rotation angles are fitted to a linear function. The exact fitting functions $r_{i j}(\theta)$, found after some trials, are determined and given in equation,

$$
r_{i j}(\theta)=\sum_{k=1}^{K} \xi_{k} \cos ^{K-k}\left(\frac{\theta}{2}\right) \sin ^{k-1}\left(\frac{\theta}{2}\right), K=2 J+1 ; i, j=1,2,3, \cdots, K
$$

where $K$ is the number of terms of the function, $\theta$ is rotation angle around $x, y$ or $z$ axis and $J$ is the value of spin (nuclear, electronic or coupled spins). $\xi_{k}$ is the coefficient of $k^{\prime}$ th term of linear fitting function which is determined by linear curve fitting process. Goodness of all fitting processes was controlled by the value $r$ which is known as regression coefficient or spectral radius, or visually on simultaneous plots of original and fitted curves. All operator matrices obtained were tested by comparing to corresponding operators obtained from Wigner-d formula [1] [2] [3] [4] [5] and operators in polynomial forms [9].

\section{Results and Discussion}

The calculations performed for each element of rotation matrices for all components of spins-5/2 to $7 / 2$ produce the operators given below. The symbols $c$ and $s$ are given in Equation 4. It should be noted, just for economy of calculations, that the rotation matrices can be symmetric or anti-symmetric and therefore calculations of only upper triangular matrices will suffice.

Explicit rotation operators for spin-5/2, 3 and $7 / 2$ are given below together with the recommended fit functions. Although all terms of each fitting function are given, most of the coefficients are found to be zero and therefore a few terms will be left as given below. For precise results, only several terms including non- 
zero coefficients should be taken in fitting processes.

\subsection{Rotation Operators of Spin-5/2}

Suggested fitting function for rotation operators:

$$
r_{i j}=\xi_{1} c^{5}+\xi_{2} c^{4} s+\xi_{3} c^{3} s^{2}+\xi_{4} c^{2} s^{3}+\xi_{5} c s^{4}+\xi_{6} s^{5}
$$

Rotation operators were given as,

$$
\begin{aligned}
& \hat{R}_{x}(\theta)= {\left[\begin{array}{cccccc}
r_{11} & i r_{12} & -r_{13} & -i r_{14} & r_{15} & i r_{16} \\
i r_{12} & r_{22} & -i r_{23} & -r_{24} & -i r_{25} & r_{15} \\
-r_{13} & -i r_{23} & r_{33} & -i r_{34} & -r_{24} & -i r_{14} \\
-i r_{14} & -r_{24} & -i r_{34} & r_{33} & -i r_{23} & -r_{13} \\
r_{15} & -i r_{25} & -r_{24} & -i r_{23} & r_{22} & i r_{12} \\
i r_{16} & r_{15} & -i r_{14} & -r_{13} & i r_{12} & r_{11}
\end{array}\right] } \\
& \hat{R}_{y}(\theta)=\left[\begin{array}{cccccc}
r_{11} & -r_{12} & -r_{13} & r_{14} & r_{15} & r_{16} \\
r_{12} & r_{22} & -r_{23} & -r_{24} & r_{25} & r_{15} \\
-r_{13} & r_{23} & r_{33} & -r_{34} & -r_{24} & r_{14} \\
-r_{14} & -r_{24} & r_{34} & r_{33} & -r_{23} & -r_{13} \\
r_{15} & -r_{25} & -r_{24} & r_{23} & r_{22} & -r_{12} \\
-r_{16} & r_{15} & -r_{14} & -r_{13} & r_{12} & r_{11}
\end{array}\right] \\
& \hat{R}_{z}(\varphi)=\left[\begin{array}{cccccc}
z_{11} & 0 & 0 & 0 & 0 & 0 \\
0 & z_{22} & 0 & 0 & 0 & 0 \\
0 & 0 & z_{33} & 0 & 0 & 0 \\
0 & 0 & 0 & z_{33}^{*} & 0 & 0 \\
0 & 0 & 0 & 0 & z_{22}^{*} & 0 \\
0 & 0 & 0 & 0 & 0 & z_{11}^{*}
\end{array}\right]
\end{aligned}
$$

The elements of operators were found as given below;

$$
\begin{array}{ll}
r_{11}=c^{5} & r_{22}=c^{5}-4 c^{3} s^{2} \\
r_{12}=\sqrt{5} c^{4} s & r_{23}=\sqrt{18} c^{2} s^{3}-\sqrt{8} c^{4} s \\
r_{13}=\sqrt{10} c^{3} s^{2} & r_{24}=\sqrt{18} c^{3} s^{2}-\sqrt{8} c s^{4} \\
r_{14}=\sqrt{10} c^{2} s^{3} & r_{25}=4 c^{2} s^{3}-s^{5} \\
r_{15}=\sqrt{10} c s^{4} & r_{33}=c^{5}-4 c^{3} s^{2}+3 c s^{4} \\
r_{16}=s^{5} & r_{34}=-3 c^{4} s+6 c^{2} s^{3}-s^{5} \\
z_{11}=\left(c^{5}-10 c^{3} s^{2}+5 c s^{4}\right)+i\left(5 c^{4} s-10 c^{2} s^{3}+s^{5}\right) \\
z_{22}=\left(c^{5}-2 c^{3} s^{2}-3 c s^{4}\right)+i\left(3 c^{4} s+2 c^{2} s^{3}-s^{5}\right) \\
z_{33}=\left(c^{5}+2 c^{3} s^{2}+c s^{4}\right)+i\left(c^{4} s+2 c^{2} s^{3}+s^{5}\right)
\end{array}
$$

\subsection{Rotation Operators of Spin-3}

Suggested fitting function for rotation operators:

$$
r_{i j}=\xi_{1} c^{6}+\xi_{2} c^{5} s+\xi_{3} c^{4} s^{2}+\xi_{4} c^{3} s^{3}+\xi_{5} c^{2} s^{4}+\xi_{6} c s^{5}+\xi_{7} s^{6}
$$

Rotation operators were found to be, 


$$
\begin{aligned}
& \hat{R}_{x}(\theta)=\left[\begin{array}{ccccccc}
r_{11} & i r_{12} & -r_{13} & -i r_{14} & r_{15} & i r_{16} & -r_{17} \\
i r_{12} & r_{22} & -i r_{23} & -r_{24} & -i r_{25} & r_{26} & i r_{16} \\
r_{13} & -i r_{23} & r_{33} & -i r_{34} & r_{35} & -i r_{25} & r_{15} \\
-i r_{14} & -r_{24} & -i r_{34} & r_{44} & -i r_{34} & -r_{24} & -i r_{14} \\
r_{15} & -i r_{25} & r_{35} & -i r_{34} & r_{33} & -i r_{23} & -r_{13} \\
i r_{16} & r_{26} & -i r_{25} & -r_{24} & -i r_{23} & r_{22} & -i r_{12} \\
-r_{17} & i r_{16} & r_{15} & -i r_{14} & -r_{13} & i r_{12} & r_{11}
\end{array}\right] \\
& \hat{R}_{y}(\theta)=\left[\begin{array}{cccccccc}
r_{11} & r_{12} & r_{13} & r_{14} & r_{15} & r_{16} & r_{17} \\
-r_{12} & r_{22} & -r_{23} & r_{24} & r_{25} & r_{26} & r_{16} \\
r_{13} & r_{23} & r_{33} & -r_{34} & -r_{35} & r_{25} & r_{15} \\
-r_{14} & r_{24} & r_{34} & r_{44} & -r_{34} & r_{24} & r_{14} \\
r_{15} & -r_{25} & -r_{35} & r_{34} & r_{33} & -r_{23} & r_{13} \\
-r_{16} & r_{26} & -r_{25} & r_{24} & r_{23} & r_{22} & r_{12} \\
r_{17} & -r_{16} & r_{15} & -r_{14} & r_{13} & -r_{12} & r_{11}
\end{array}\right] \\
& \hat{R}_{z}(\varphi)=\left[\begin{array}{cccccccc}
z_{11} & 0 & 0 & 0 & 0 & 0 & 0 \\
0 & z_{22} & 0 & 0 & 0 & 0 & 0 \\
0 & 0 & z_{33} & 0 & 0 & 0 & 0 \\
0 & 0 & 0 & z_{44} & 0 & 0 & 0 \\
0 & 0 & 0 & 0 & z_{33}^{*} & 0 & 0 \\
0 & 0 & 0 & 0 & 0 & z_{22}^{*} & 0 \\
0 & 0 & 0 & 0 & 0 & 0 & z_{11}^{*}
\end{array}\right]
\end{aligned}
$$

The elements of operators were found as given below;

$$
\begin{aligned}
& r_{11}=c^{6} \\
& r_{24}=\sqrt{30}\left(c^{4} s^{2}+c^{2} s^{4}\right) \\
& r_{12}=\sqrt{6} c^{5} s \\
& r_{25}=\sqrt{40} c^{3} s^{3}-\sqrt{10} c s^{5} \\
& r_{13}=\sqrt{15} c^{4} s^{2} \\
& r_{26}=5 c^{2} s^{4}+s^{6} \\
& r_{14}=\sqrt{20} c^{3} s^{3} \\
& r_{33}=c^{6}-8 c^{4} s^{2}+6 c^{2} s^{4} \\
& r_{15}=\sqrt{15} c^{2} s^{4} \\
& r_{34}=\sqrt{108} c^{3} s^{3}-\sqrt{12}\left(c^{5} s+c s^{5}\right) \\
& r_{16}=6 c s^{5} \\
& r_{35}=-6 c^{4} s^{2}+8 c^{2} s^{4}-s^{6} \\
& r_{17}=s^{6} \\
& r_{44}=c^{6}-9\left(c^{4} s^{2}+c^{2} s^{4}\right)-s^{6} \\
& r_{22}=c^{6}-5 c^{4} s^{2} \\
& r_{23}=-\sqrt{10} c^{5} s+\sqrt{40} c^{3} s^{3} \\
& z_{11}=\left(c^{6}-15 c^{4} s^{2}+15 c^{2} s^{4}-s^{6}\right)+i\left(6 c^{5} s-20 c^{3} s^{3}+6 c s^{5}\right) \\
& z_{22}=\left(c^{6}-5 c^{4} s^{2}-5 c^{2} s^{4}+s^{6}\right)+4 i\left(c^{5} s-c s^{5}\right) \\
& z_{33}=\left(c^{6}+c^{4} s^{2}-c^{2} s^{4}-s^{6}\right)+2 i\left(c^{5} s+2 c^{3} s^{3}+c s^{5}\right) \\
& z_{44}=1
\end{aligned}
$$

\subsection{Rotation Operators of Spin-7/2 (Octet)}

Suggested fitting function for rotation operators:

$$
r_{i j}=\xi_{1} c^{7}+\xi_{2} c^{6} s+\xi_{3} c^{5} s^{2}+\xi_{4} c^{4} s^{3}+\xi_{5} c^{3} s^{4}+\xi_{6} c^{2} s^{5}+\xi_{7} c s^{6}+\xi_{8} s^{7}
$$


Rotation operators are given as,

$$
\begin{aligned}
& \hat{R}_{x}(\theta)=\left[\begin{array}{cccccccc}
r_{11} & i r_{12} & r_{13} & i r_{14} & r_{15} & i r_{16} & r_{17} & r_{18} \\
i r_{12} & r_{22} & i r_{23} & r_{24} & i r_{25} & r_{26} & i r_{27} & r_{17} \\
r_{13} & i r_{23} & r_{33} & i r_{34} & r_{35} & i r_{36} & r_{26} & i r_{16} \\
i r_{14} & r_{24} & i r_{34} & r_{44} & i r_{45} & r_{35} & i r_{25} & r_{15} \\
r_{15} & i r_{25} & r_{35} & i r_{45} & r_{44} & i r_{34} & r_{24} & i r_{14} \\
i r_{16} & r_{26} & i r_{36} & r_{35} & i r_{34} & r_{33} & i r_{23} & r_{13} \\
r_{17} & i r_{27} & r_{26} & i r_{25} & r_{24} & i r_{23} & r_{22} & i r_{12} \\
i r_{18} & r_{17} & i r_{16} & r_{15} & i r_{14} & r_{13} & i r_{12} & r_{11}
\end{array}\right] \\
& \hat{R}_{y}(\theta)=\left[\begin{array}{cccccccc}
r_{11} & r_{12} & r_{13} & -r_{14} & r_{15} & r_{16} & -r_{17} & -r_{18} \\
-r_{12} & r_{22} & r_{23} & -r_{24} & -r_{25} & r_{26} & r_{27} & -r_{17} \\
r_{13} & -r_{23} & r_{33} & r_{34} & -r_{35} & -r_{36} & r_{26} & r_{16} \\
r_{14} & -r_{24} & -r_{34} & r_{44} & r_{45} & r_{35} & -r_{25} & r_{15} \\
r_{15} & r_{25} & r_{35} & -r_{45} & r_{44} & r_{34} & -r_{24} & -r_{14} \\
-r_{16} & r_{26} & r_{36} & -r_{35} & -r_{34} & r_{33} & r_{23} & r_{13} \\
-r_{17} & -r_{27} & r_{26} & r_{25} & -r_{24} & -r_{23} & r_{22} & r_{12} \\
r_{18} & r_{17} & -r_{16} & r_{15} & r_{14} & r_{13} & -r_{12} & r_{11}
\end{array}\right] \\
& \hat{R}_{z}(\varphi)=\left[\begin{array}{cccccccc}
z_{11} & 0 & 0 & 0 & 0 & 0 & 0 & 0 \\
0 & z_{22} & 0 & 0 & 0 & 0 & 0 & 0 \\
0 & 0 & z_{33} & 0 & 0 & 0 & 0 & 0 \\
0 & 0 & 0 & z_{44} & 0 & 0 & 0 & 0 \\
0 & 0 & 0 & 0 & z_{44}^{*} & 0 & 0 & 0 \\
0 & 0 & 0 & 0 & 0 & z_{33}^{*} & 0 & 0 \\
0 & 0 & 0 & 0 & 0 & 0 & z_{22}^{*} & 0 \\
0 & 0 & 0 & 0 & 0 & 0 & 0 & z_{11}^{*}
\end{array}\right]
\end{aligned}
$$

The elements of operators were found as given below;

$$
\begin{array}{rlrl}
r_{11} & =c^{7} & r_{12} & =\sqrt{7} c^{6} s \\
r_{13} & =\sqrt{21} c^{5} s^{2} & r_{14} & =\sqrt{35} c^{4} s^{3} \\
r_{15} & =\sqrt{35} c^{3} s^{4} & r_{16} & =\sqrt{21} c^{2} s^{5} \\
r_{17} & =\sqrt{7} c s^{6} & r_{18} & =s^{7} \\
r_{22} & =c^{7}-6 c^{5} s^{2} & r_{23} & =-\sqrt{12} c^{6} s+\sqrt{75} c^{4} s^{3} \\
r_{24} & =-\sqrt{45} c^{5} s^{2}+\sqrt{80} c^{3} s^{4} & r_{25} & =\sqrt{80} c^{4} s^{3}-\sqrt{45} c^{2} s^{5} \\
r_{26} & =\sqrt{75} c^{3} s^{4}-\sqrt{12} c s^{6} & r_{27} & =-6 c^{2} s^{5}+s^{7} \\
r_{33} & =c^{7}-10 c^{5} s^{2}-c^{3} s^{4} & r_{34} & =\sqrt{15} c^{6} s-\sqrt{240} c^{4} s^{3}+\sqrt{60} c^{2} s^{5} \\
r_{35} & =\sqrt{60} c^{5} s^{2}-\sqrt{240} c^{3} s^{4}+\sqrt{15} c s^{6} & r_{36} & =10 c^{3} s^{4}-10 s^{5} c^{2}+s^{7} \\
r_{44} & =c^{7}-12 s^{2}+18 c^{3} s^{4}-4 c s^{6} & r_{45} & =4 c^{6} s-18 c^{4} s^{3}+12 c^{2} s^{5}-s^{7} \\
z_{11} & =\left(c^{7}-21 c^{5} s^{2}+35 c^{3} s^{4}-7 c s^{6}\right)+i\left(7 c^{6} s-35 c^{4} s^{3}-21 c^{2} s^{5}-s^{7}\right) \\
z_{22} & =\left(c^{7}-9 c^{5} s^{2}-5 c^{3} s^{4}+5 c s^{6}\right)+i\left(5 c^{6} s-5 c^{4} s^{3}-9 c^{2} s^{5}+s^{7}\right) \\
z_{33}=\left(c^{7}-c^{5} s^{2}-5 c^{3} s^{4}-3 c s^{6}\right)+i\left(3 c^{6} s+5 c^{4} s^{3}+c^{2} s^{5}-s^{7}\right) & \\
z_{44}=\left(c^{7}+3 c^{5} s^{2}+3 c^{3} s^{4}+c s^{6}\right)+i\left(c^{6} s+3 c^{4} s^{3}+3 c^{2} s^{5}+s^{7}\right)
\end{array}
$$




\section{Conclusions}

The electron spin can form a dual thread, the total spin is 1 , and it can form a triplet state or qutrite system. In addition, carbon nanotubes or fullerens will enable three or more paired electron systems. Therefore, it seems necessary to establish the theoretical foundations of large spin systems. Since EPR spectroscopy can work in different spin systems, quantum mechanical rotation for some systems other than spin $1 / 2$, some basic passages and spin processors need to be created. Quantum rotation processors for spin 5/2,3 and 7/2 were found in this study.

Quantum mechanical rotation operators in explicit forms were constructed by numerical evaluations of exponential rotation operator for spins-5/2 to $7 / 2$. The exponential rotation operator was expanded in power series and numerical values for each operator element at angles consequently between definite intervals, and then the values were fitted to linear trigonometric polynomials of sines and cosines. The rotation operators obtained can be used to determine rotations of corresponding spin around three Cartesian coordinates.

\section{Conflicts of Interest}

The authors declare no conflicts of interest regarding the publication of this paper.

\section{References}

[1] Wigner, E.P. (1959) Group Theory and Its Applications to the Quantum Mechanics of Atomic Spectra. Academic Press, Los Alamos.

[2] Messiah, A. (1967) Quantum Mechanics. Vol. 2, North Holland Publishing Co., Orsay.

[3] Sakurai, J.J. and Napolitano, J.J. (2011) Modern Quantum Mechanics. Cambridge University Press, Cambridge.

[4] Morrison, M.A. and Parker G.A. (1987) A Guide to Rotations in Quantum Mechanics. Australian Journal of Physics, 40, 465-498.

https://doi.org/10.1071/PH870465

[5] Blanca, M.A., Flórez, M. and Bermejo, M. (1997) Evaluation of the Rotation Matrices in the Basis of Real Spherical Harmonics. Journal of Molecular Structure: THEOCHEM, 419, 19-27. https://doi.org/10.1016/S0166-1280(97)00185-1

[6] Dachsel, H. (2006) Fast and Accurate Determination of the Wigner Rotation Matrices in the Fast Multipole Method. Journal of Chemical Physics, 124, Article ID: 144115. https://doi.org/10.1063/1.2194548

[7] Gimbutas, Z. and Greengard, L. (2009) A Fast and Stable Method for Rotating Spherical Harmonic Expansions. Journal of Computational Physics, 228, 5621-562. https://doi.org/10.1016/j.jcp.2009.05.014

[8] Aubert, G. (2013) An Alternative to Wigner D-Matrices for Rotating Real Spherical Harmonics. AIP Advances, 3, Article ID: 062121. https://doi.org/10.1063/1.4811853

[9] Curtright, T.L. Fairlie, D.B. and Zachos, C.K. (2014) mCompact Formula for Rotations as Spin Matrix Polynomials. Symmetry, Integrability and Geometry: Methods and Applications, 10, 1-15. https://doi.org/10.3842/SIGMA.2014.084 
[10] Curtright, T.L. and Van Kortryk T.S. (2014) On Rotations as Spin Matrix Polynomials. Journal of Physics A: Mathematical and Theoretical, 48, Article No. 025202. https://doi.org/10.1088/1751-8113/48/2/025202

[11] Fukushima, E. and Roeder, S.B.W. (1993) Experimental Pulse NMR: A Nuts and Bolts Approach. CRC Press, Boca Raton.

[12] Rule, G.S. and Kelvin Hitchens, T. (2006) Fundamentals of Protein NMR Spectroscopy. Springer, Netherlands.

[13] Oliviera, I., Sarthour Jr., R., Bonagamba, T., Azevedo, E. and Freitas, J.C.C. (2007) NMR Quantum Information Processing. Elsevier, Amsterdam.

[14] Jones, J.A. (2001) NMR Quantum Computation. Progress in Nuclear Magnetic Resonance Spectroscopy, 38, 325-360. https://doi.org/10.1016/S0079-6565(00)00033-9

[15] Schweiger, A. and Jeschke, G. (2001) Principles of Pulse Electron Paramagnetic Resonance. Oxford University Press, Oxford. 